\title{
XIV. On crystallography. Translated from the last Paris edition of his Traité de Minéralogie
}

\section{Hauy}

To cite this article: M. Hauy (1809) XIV. On crystallography. Translated from the last Paris edition of his Traité de Minéralogie, Philosophical Magazine Series 1, 34:136, 96-108, DOI: $10.1080 / 14786440908562932$

To link to this article: http://dx.doi.org/10.1080/14786440908562932

曲 Published online: 18 May 2009.

Submit your article to this journal $\sqsubset \pi$

Џ Article views: 2

Q View related articles $\square$ 


\section{[ 966 ]}

XIV. On Crystallography. By M. Haux. Translated fiom the last Paris Edition of his Traité de Mineralogie.

[Continued from p. 69.]

\section{OF CRYSTALLIZATION.}

$W_{E}$ have been led by observation and reasoning to ascertain that minerals were cọmposed of similar integrant molecules. The way in which they are mechanically divided has besides proved to us, that the canse which solicited these molecules to be mutually attracted; reunited them in rows upon planes situated in the direction of their different faces. These considerations were useful for preparing the developinent of another very remarkable result of the laws of affinity, which is referred to the external configuration of minerals, and has given birth to one of the most fertile branches of the science of which they are the objects.

Such therefore is the action of these laws upon integrant molecules, that, when nothing disturbs it, the assemblages of these molecules are terminated by plane surfaces, whence result regular forms similar to those of geometrical solids. We have frequent examples of this regularity in the garnet, topaz, emerald, carbonated lime, sulphated barytes, \&c.; and in a great number of metallic substances. The sight of these polyhedrons always excites the surprise of a person to whom they are for the first time presented, and they must frequently be shown to him incrusted with their native earth before he can believe in the geometry of nature.

All these regular bodies have been received under the common denomination of crystals*. The term crystallization, which would at first sight appear to apply only to the species of operation from which crystals are produced, has generally a more extehsive signification. It expresses in general every assemblage of molecules in solid masses by the help of affinity. If these masses have a symmetrical aspect, they will be the products of regular crystallization properly so called. If their form is vague, and cannot be determined in a precise manner, they will belong to confused crystallization.

The attractive forces which solicit the molecules of a mineral, suspended in a liquid, have a certain reference with the figure of these molecules, and it is in this relation that the tendency consists which the molecules have of themselves to unite conformably to the laws of a regular aggregation. But in order that they may attain this object, they

* The origin of this word will be found under the article of Quartz. 
must have leisure to seek each other, to apply to each uther by proper surfaces, and to concur all at the same time to the harmony which ought to result from their aggregation. The liquid must be in a state of repuse; its own molecules must slowly abandon those of the mineral, in order to place them in the position most favourable to affinity, and the cavity must be spacious enough, and the liquid sufficiently abundant, for the crystalline molecules to swim in it at full liberty.

If these conditions are not fulfilled; if it happens, for instance, that the liquid is rapidly evaporated, or that any agitation is produced in it, these accidents, which we may regard as disturbing causes of crystallization, will in some measure disarrange the molecules, and force them to be tumultuously precipitated upon each other; and, as a necessary consequence, the traces of the geometrical form which would have taken place in the event of a slow and tranquil aggregation will he more or less altered *.

But as we are only to speak here of crystallization properly 20 called, an important consideration in the first place presents itself, $-\mathrm{a}$ consideration which places, minerals by the side of organic beings. In the vegetable kingdom, for instance, all the individuals of the same species seem to have been made after one common model, $i$. e. their flower is composed of parts equal in number and similar in figure; their leaves have the same arrangement, the same contours, \&c. - the diversities consist of but light and fugitive shades. In short, when we have seen a single plant, we have seen the whole species.

It is totally different with respect to minerals. Frequently crystals originating from one and the same substance assume very different forms, all equally distinct, and executed with

\footnotetext{
* The crystals formed in one and the same liquid around different centres of action more or less closely connected with each other, compose groups in which they are situated sometimes parallel to each other, and sometimes crossing each other in different directions, in such a way that they frequently enough appear to penetrate each other mutually. It also happens very gene. rally that they are only salient at one of their parts, above the substance which serves as a support to them. It is a fortunate circumstance when a crystal belongs to the group only by a point, so that its position isolate it in some messure, and permits its form to be entirely developed to the eyes of an observer. But most of the crystals which offer this advantage have been extracted from certain earthy masses in which they were really solitary, and in the nidst of which they are formed at the time when these earths were diluted in an aqueous fluid. We may conceive this formation from an experiment of Pelletier, who, having placed argil soaked in a solution of alum, cut this argil into pieces when it was dry, and found internally crystals of alum of the size of a pea. Hence he concluded that the crystalline molecules might have had the power of displacing the argillaceous molecules, and of removing those bstacles which opposed their union.-Mem. et Observ. de Chimie, t. i. p. 81.
}

Vol.34. No. 136. August 1809. 
similar precision. Carbonated lime, for instance, assumes, according to circumstances, the form of a rhomboid; of a regular hexahedral prism; of a solid terminated by twelve scalene angles; or of a dodecabedron with pentagonal faces, \&c. Sulphuretted iron, or ferruginous pyrites, produces sometimes cubes and sometimes regular octahedrons; here dodecahedrons with pentagonal faces, there icusahedrons with triangular faces, \&c.

It is true that, among the varieties of one and the same species, it often happens that a more compound form differs from a more simple form only by certain facets, similar to those which would result from sections made on the solid angles, or on the ridges of the latter*. Pyrites, for instance, sometimes assumes the form of a cube, the eight solid angles of which being beaten down would expose to view so many triangular facets, in such a manner that this form may be considered as the passage from the cube to the octahedron, with which it is connected by its eight equilateral triangles, which are situated like the faces of this second solid.

But in addition to these transitions being already very singular in themselves, as appertaining to modifications much more sensible than seems to be necessary to distinguish simple varieties, we find on the other hand certain crystalline forms, which, by a singularity still more remarkable, do not exhibit any vestiges of common parts, and present the appearance of a complete metamorphose of the mineral from which they derive their origin. And in order to cite a new example, let us place by the side of each other the regular hexahedral prism of carbonated lime, (Pl.I., fig. 1.) and the scalene dodecahedron with scalene triangular faces (fig. 6.). We can scarcely conceive how two polyhedrons, so dissimilar at first view, come to touch together, and are as if confounded in the crystallization of one and the same mineral.

In short, as if the results of this operation of Nature were destined to excite astonishment of every kind, while one and the same substance lends itself to so many transformations, we meet very different substances, which present absolutely the same form. Thus fluated lime, muriated soda, sulphuretted iron, sulphuretted lead, \&c., crystallize in cubes under certain circumstances; and in other cases, the same

* This idea suggested to M. Romé de l'Isle the method of truncatures, for enabling us to derive from each other the different varieties of crystalline forms which should belong to one and the same substance.

minerals, 
minerals, as well as sulphated alumine and the diamond, assume the furm of a regular octahedron *.

It was this similitude of forms which, at a time when the study of crystallization was scarcely in its infancy, inclined Liunæus to think that the salts should be regarded as the generators of crystallization; that the union of any given salt with a given kind of stone was a sort of fecundation which communicated to the stone the property of crystallizing under the form peculiar to the salt which performed the function of the fecundating principle + . The diamond, for instance, be considered as a species of alum, because it crystallizes like the latter, and he gave it the name of alumen adamas $\ddagger$. Thus Linnæus thought he found in the mineral kingdom the basis of the sexual system; of which he made so advantageous an use in Botany. We know that Tournefort, on observing the ramified stalactites of the Grotto of Antiparos, imagined that stones vegetated in the same way as plauts. Botany was the favourite study of these two ces lebrated men, and all Nature, in their opinion, spoke the language of their favourite study.

Linnæus subjoined to his work some descriptions and figures of crystals, which were sufficiently accurate, considering the state of science at the time; and in this respect he may be regarded as the founder of crystallography.

Latterly, Romé de l'Isle has referred the study of cry* stallization to principles more and more conformable to observation. He arranged together, as far as possible, crystals of the same nature. Among the different forms relative to each species, he chose one as the most proper, from its simplicity, to be regarded as the primitive form; and by supposing it truncaled in different ways, he deduced the other forms from it, and determined a gradation, a series of transitions between this same form and that of polyhedrons, which seemed to be still further removed from it. To the descriptions and figures which he gave of the crystalline forms, he added the results of the mechanical measurement

* We shall presently explain the reasons which can assist us in conceiving the nature of this resemblance in configuration between minerals of various descriptions.

+ Linnai Amoenit. Acad. tome i. p. 466 \& seq.

The learned author of this classification was well aware that, among the bodies which he associated under one and the same species, several presented a form different from that which was the type of the species. Bnt he tried to bring them to this last form according to some vague traces of resemblance which he caught from their external aspect; and as but a very small number of crystalline forms had been at that time observed; the most of them extremely simple, these similarities, which would have been impracticable in the present advanced state of science, were then of less difficult classification. 
of their principal angles, and showed (what was a very essential point) that these angles were constant in each variety. In a word, his crystallography is the production of a very extensive and highly usefirl course of study.

The illustrious Bergman, by endeavouring to penetrate to the mechanism of the structure of crystals, considered the different forms relative to one and the same substance as produced by a superposition of planes, sometimes constant and sometimes variable, and decreasing around one and the same primitive form. He applied this primitive idea to a small number of crystalline forms, and verified it with respect to a variety of calcareous spar* by fractures, which enabled him to ascertain the position of the nucleus, or of the primitive form, and the successive order of the lamina covering this nucleus. Bergman, however, stopped here, and did not trouble himself either with determining the laws of structure, or applying calculation to it. It was a simple sketch, drawn en passant, of the most prominent point of view in mineralogy, but in which we see the hand of the same master who so successfully filled up the outlines of chemistry.

In the researches which I undertook about the same period on the structure of crystals $t$, I proposed combining the form and dimensions of integrant molecules with simple and regular laws of arrangement, and submitting these laws to ealculation. This work produced a mathematical theory, which I reduced to analytical formuls, representing every possible case, and the application of which to known forms leads to valuations of angles constantly agreeing with observation. I shall explain the principles of this theory by the help of reasoning alone, and of some projertions which will facilitate their explanation. Geometricians may acquire a more correct and more detailed knowledge of the subject by perusing the ealculations which are given separately in this work.

\section{THEORY OF THE STRUCTURE OF CRYSTALS:}

Primitive Forms.- The idea of referring to one of the same primitive forms all the forms which may be assumed by a mineral substance, of which the rest may be regarded as being modifications only, has frequently suggested itself to various naturalists who have made crystallography their study. It was in consequence of having regarded it in a false point.

* This is what has been called dent de cochon, but which I call metostafic.

t The members of the Academy of Sciences were acquainted with my first Fssays on this subject, when they received Bergman's Memoir, which was communicated to me as being interesting. 
of view that Linnæus was led astray in his methodical dis. tribution of crystals. Romé de l'Isle, by employing it with more art and justice, avoided the breaches of natural connections which disfigure the system of the Swedish naturalist. But there was something arbitrary in the choice of the forms which De l'Isle regarded as primitive, by consulting only the external aspect of crystals, without regarding their structure. Bergman, who had so successfully seized the nucleus of carbonated lime, by mechanically dividing the metastatic crystal, was not equally fortunate with respect to several other crystals, and in particular with respect to the dodecahedron variety of the same substance which was then called tête de clou (nail head). It would result from the explanation which he gives of the structure of this crystal, that its nucleus should have angles totally different from those of the true; and Bergman has even been obliged to suppose that the planes which he calls fundamental were truncated in the present case, which presented a new exception to the principle on which his system was founded*.

The mechanical division of minerals, which is the only method of ascertaining their true primitive form, proves that this form is invariable while we operate upon the same substance, however diversified or dissimilar the forms of the crystals belonging to this substance may be. Two or three examples will serve to place this truth in its proper light.

Take a regular hexahedral prism of carbonated lime (PI. I. figs. 1 and 2 ). If you try to divide it parallel to the edges which form the contours of the bases, you will find that three of these edges taken alternately in the upper part, for instance, the edges $l f, c d, b m$, may be referred to this division: and in order to succeed in the same way with respect to the inferior base, we must choose, not the edges $l^{\prime} f^{\prime}, c^{\prime} d^{\prime}, b^{\prime} m^{\prime}$, which correspond with the preceding, but the intermediate edges $d^{\prime} f^{\prime}, b^{\prime} c^{\prime}, l^{\prime} m^{\prime}$.

The six sections will uncover an equal number of trapeziums. Three of the latter are represented upon fig. 2 , viz. the two which intercept the edges $l f, c d$, and are designated by $p \rho 00$, a $a k$, and that which intercepts the lower edge $d^{\prime} f^{\prime}$, and which is marked by the letters $n n i i$.

Each of these trapeziums will have a lustre and polish, from which we may easily ascertain that it coincides with one of the natural joints of which the prism is the assemblage. You may attempt in vain to divide the prism in any other direction. But if you continue the division parallel

* See the remarks on this head in my Essty towards a Theory of the Structure of Crystals,-See Philosoplical Magazine, vol. $i_{\text {. }}$ p. $3 s$ et seq. 
to the first sections, it will happen that on one hand the surfaces of the bases will always become narrower, while on the other hand the altitudes of the panes will decrease; and at the term at which the bases have disappeared, the prism will be changed into a dodecahedron (fig. 3 ,) with pentagonal faces, six of which, such as o o $i$ O $e, 0 I k i i$, \&c., will be the residues of the panes of the prism; and the six others EA Ioo, $\mathrm{OA}^{\prime} \mathrm{K} i \mathrm{i}$, \& $\mathrm{c}$. will be the immediate result of the mechanical division *.

Beyond this same term, the extreme faces will preserve their figure and dimensions, while the lateral faces will incessantly diminish in height, until the points $o, k$, of the pentagon $\theta$ I $k i i$, coming to be confounded with the points $i, i$, and so on with the other points similarly situated, each pentagon will be reduced to a simple triangle, as we see in fig. $4 .+$

Lastly, when new sections have obliterated these triangles, so that no vestige of the surface of the prism remains, (fig. 1 ,) you will have the nucleus or the primitive form, which will be an obtuse rhomboid $\downarrow$, (fig. 5,) the grand angle of which EA I or EOI is $101^{\circ} 32^{\prime} 13^{\prime \prime} \S$.

The observation I have detailed is that which served for developing $m y$ ideas on the structure of crystals, and has been the key of the theory: it occurred to me on the occasion of a crystal being presented to me by citizen Defranee from his mineralogical collection. The prism had a single fracture at the place of one of the edges situated around the base, by which it had adhered to the remains of the group. Instead of placing it in my collection, I tried to divide it in other directions; and I succeeded after some

* We have continued to represent the hexahedral prism circumscribed by the solid from which we extract it, by dividing it, in order that the progress of the operation may be more easily conceived.

$\uparrow$ The points which are confounded, two and two, upon this figure are each marked with the two letters which served to designate them when they were separated, as in fig. 3.

$\neq$ I denominate as a thomioid a parallelopipedon, terminated by six equal and similar thombuses. Two of the solid angles, such as $A, A^{\prime}$, opposed to each other, are formed by the union of three equal plane angles. Each of the six others is formed by a plane angle equal to the preceding ones, and by two angles which are supplements to them. The points $A, A^{\prime}$, are the summits, and the line which proceeds from the one to the other is the axis. We always suppose the rhomboid situated so as to make its axis vertical, In any single face, such as $A E O I$, the line drawn from $E$ to $I$ is the horizontal diagonal, and that from $A$ to $Q$ is the oblique diagonal. The rhomboid is obtuse or acute, accordingly as the angle contiguous to the summit is itself obtuse or achte.

\$ I have observed that each trapezium, such as $p p$ oo (fig. 2,) uncovered by the first sections, was very sensibly inclined from the same quantity, as well upon the residue $p p$ de $l m$ of the base, as upon the residue o o $f^{\prime} l^{\prime}$ of the adjacent pane. Setting out from this equality of inclinations, we deduce from it by calculation the value of the angles with the precision of minutes and seconds, which mechanical measurements are not capable of attaining. 
inefiectual attempts in extracting its rhomboidal nucleus; which excited my surprise, mixed with the hope that I might succeed still further.

Let us take as a second example-the metastatic crystal (fig: 6, the nucleus of which was found by Bergman. You may obtain this nucleus speedily by making a first section upon the elges EO, OI; a second upon the edges I K, GK ; a third upon $\mathrm{GH}, \mathrm{EH}$; a fourth upon $\mathrm{OI}, \mathrm{IK}$; a fifth upon $\mathrm{GK}, \mathrm{GH}$; lastly, a sixth upon $\mathrm{EH}, \mathrm{EO}$; whence it follows, that the edges of which we are speaking are confounded with the lateral edges of the primitive form, as we may judge from a simple inspection of fig. 7 , which represents this primitive form inscribed in the dodecahedron with scalene triangles.

There exist many other varieties of carbonated lime, several of which have very compound forms, and all of them contain a nucleus precisely similar to that under consideration. But if it be singular to set this nucleus issue from varieties which are removed from it mostly by their configuration, we have far less reason to expect it in those which of themselves have a rhomboidal form with different measurements of angles. We are at present acquainted with five of these rhomboids*, one of which is much more obtuse than the nucleus, and the four others have summits always more acute. This gradation, all the terms of which are referred to one and the same species of solid, would seem at first sight to give some colour to the opinion that prinitive forms are not constant relative to one and the same mineral. But $I$ have ascertained that all these rhomboids concur in exhibiting, by sections made in different directions, a nucleus sinilar to that whose grand angle is $101^{\circ} 32^{\prime}$; and thus the paradox which arises from the diversity of their angles is cleared up by the double employment

* Beginning with that which is obtuse, we have the following values for the plane angle of the summit:

$\begin{array}{ccc}114^{0} & 18^{\prime} & 56^{\prime \prime} \\ 87 & 42 & 30 \\ 75 & 31 & 20 \\ 45 & 34 & 22 \\ 37 & 31 & 4\end{array}$

Mineralogists for a long period have only been acquainted with the first and third of these rhomboids. We see from Bergman's memoir, which I have already quoted, that he confounded the rhomboid $114^{\circ} 18^{\prime}$ with the pri: mitive, in which the angle of the summits is $10^{\circ} 32^{\prime}$. On the other hand; Romé de l'Isle considered that of $75^{\circ} 31^{\prime}$ as a second primitive form of calcareous spars, because he saw no method of referring it, even by truncatures, to that of $101^{\circ} 32^{\prime}$. See Bergman's work on the character of minerals. Tableau Lithologique. 
of the rhomboidal form which serves here to disguise itself, and conceals fixed characters under variable outsides.

Let us choose for instance, among these different rhomboids, that in which the angle at the summit is $75^{\circ} 31^{\prime} 90^{\prime \prime}$, and which is represented fig. 8 , circumscribed to its nucleus. Romé de l'Isle called it muriatic calcareous spar, and I denominate it inverse carbonated lime. In order to divide this rhomboid mechanically, the secting planes must be directed parallel to the six extreme edges; viz. $s t, s u, s n$, on one hand, and $s t^{\prime}, s u^{\prime}, s n^{\prime}$, on the other, in such a way that these planes are equally inclined upon the faces which they cut into. The first sections will exhibit six pentagons $r r r r^{\prime} r^{\prime} r^{\prime}$ (fig. 9), parallel to the faces of the nucleus; and it is easy to conceive, that by continuing the division always in the same direction, until the residues of the faces of the rhomboid A $A^{\prime}$ (fig. 8) have disappeared, we shall have a new rhomboid, which will be the primitive form.

We may remark, that the faces of this last rhomboid are inclined in the same quantity upon the common axis, with the pdges $s t, s u, s . n$, \&xc.; to which these faces are parallel. Now the edges in question form with the axis larger angles than the oblique diagonals drawn from $s$ to $n^{\prime}$, from $s$ to $t^{\prime}$, from $s$ to $u^{\prime}$, or, what comes to the same thing, than the faces $s t n^{\prime} u$, snt $t^{\prime} u, s t u^{\prime} n$; whence we conclude that in the rhomboid, extracted by mechanical division, the angle of the summit should be sensibly more open than that which corresponds with it in the divided rhomboid. From what has been said above, this last angle is smaller than the other by $26^{\circ}$ of $53^{\prime \prime}$.

If we try to divide a crystal of another species, you will have a different nucleus. For instance, a cube of fluated lime will give a regular octahedron, which you will succeed in extracting by dividing the cube upon its eight solid angles, which will in the first place discover eight equilateral triangles, and by pursuing the division, always parallel to the first sections, until nothing more remains of the faces of the cube, the nucleus of the crystals of sulphated barytes will be a straight prism with rhombous bases; that of the crystals of phosphated lime a regular hexahedral prism; that of sulphuretted lead a cube, \&c.; and each of these forms will be constant relative to the entire species, in such a manner that its angles will not undergo any appreciable variation.

With regard to crystals which refuse to be mechanically divided, the theory seconded by certain indications, which 
we shall presently speak of, may lead to the determindtion of their primitive forms, at least with a great probability of accuracy.

Having adopted the word primitive form, in order to designate the nucleus of crystals, we shall call secondary forms such varieties as differ from the primitive form.

In certain species, crystallization also produces this last form immediately. There exist, for example, calcareous crystals which differ in no respect from the rhomboid which we extrect from the regular hexahedral prism, and from the other varieties which we have mentioned. Besides, it frequently happens also, that among the faces of a secondary crystal there are some which are parallel to those of the primitive form. Thus we find crystals of carbonated lime, which are similar to that of fig. 3 , and on which crystallization has left pentagonal planes, such as A E oo I, A E $h h \mathrm{G}$, \&c., situated like those which we expose by dividing the hexahedral prism represented in fig. 1 . In such cases as these, the route is as it were traced out before hand, previous to arriving at the nucleus.

We may define the primitive form a solid of a canstant form, engaged symmetrically in all the crystals of one and the same species, and the faces of which follow the directions of the laminæ which form these crystals.

The primitive forms hitherto observed are reduced ta six, viz.: the parallelopipedon, the octahedron, the tetrahedron, the regular hexahedral prism, the dodecahedron with rhombous planes, all equal and similar, and the dodecahedron with triangular planes, composed of two straight pyramids joined base to base.

Forms of integrant Molecules. - The nucleus of a crystal is not the last term of its mechanical division. It may always be subdivided parallel to its different faces, and sometimes in other directions also. The whole of the surrounding substance is capable of being divided by strokes parallel to those which take place with respect to the primitive form. Reasoning here as we have done with respect to the mechanical division of common salt, we conclude that the limit of the mechanical division which we may operate in any given crystal, ought to give the form of the integrant molecule peculiar to the kind of mineral of which this crystal is the primitive.

If the nucleus be a parallelopipedon which cannot be subdivided except by blows parallel to its faces, like that which takes place with respect to carbonated lime, it is evident that the integrant molecule will be similar to this nucleus itself.

But it may happen that the parallelopipedon admits of further 
further sections in other directions than the former. Let us conceive for example, that it is a rhomboid $\mathrm{A} \mathrm{A}^{\prime} \mathrm{K} \mathrm{H}$ (fig.10), directly divisible paralle! to the six rhombuses which terminate it, and with the help of planes, each of which passes by an oblique diagonal $A O$, by the axis $A^{\prime} A$, and by' the edge $A^{\prime} O$, comprehended between the same diagonal and the axis. These sections will detach six tetrahedrons, which have been figured separately around the rhomboid, in positions analogous to those which they had when joined in one single body, in such a way that we follow as it were with the eye the species of decomposition of the rhomboid from wbich they proceed. Now these tetraliedrons represent the integrant molecules of the substance of which the rhomboid is the primitive form. Such is the structure of the tourmaline.

Let us take another substance, such as phosphated lime, the primitive form of which is the regular hexahedral prism. In this case the molecule will be still different from the-nucleus, although this last cannot be subdivided except parallel with its faces, $i$. e. with its two bases and its six panes. This subdivision will, lead us to triangular prisms, the assemblage of which composes the entire prism, ; as may easily be perceived by inspecting fig. $40, \mathrm{Pl}$. V; we there see one of the bases of the prism divided inta equilateral triangles, each of which is the base of a small triangular prism which represents the integrant molecule.

Now we shall presently find that we may reduce the forms of the integrant molecules of all crystals to the three preceding forms, which are the tetrahedron, or the simplest of the pyramids : the triangular prism, or the simplest of all the prisms; and the parallelopipedon, or the simplest among the solids, which have their faces parallel two and two. And since four planes at least are necessary for circumscribing a space, it is evident that the three forms in question, in which the number of faces is successively four, five, and six, have still, in this respect, the greatest possible simpliwity. If these forms, I repeat, are not those of the true integrant molecules employed by Nature, they deserve at least to supply their place in our conceptions, the more especially as it is with but very scanty materials that we succeed in establishing a theory which embraces so many various results.

Several naturalists have thought that the integrant molecules of crystals were simple lamina, whose thickness was incomparably less than their other dimensions, and not small solids, the thickness of which was equal, or at least in proportion to their breadth and length. In the Journal dos Mines, No. 28, p. 305, I have detailed the numerous 
and decisive proofs which confirm this last opinion, and I have even demonstrated that the dimensions and angles of these molecules were invariable in all primitive crystals of one and the same substance. I shall not stop here again to obviate the difficulties which opposed my progress, because I have had the satisfaction of observing that the answers suggested themselves as if spontaneously to those who are in posession of the theory, and because it appears to me that this theory has been taken up by the only author who studied it in the same points of view with myself*.

But every form of the integrant molecule varies in its dimensions, or in the measurement of its angles, according to the species to which it belongst. The parallelopipedon is sometimes oblique-angled, and sometimes right-angled: sometimes it presents the form of the rhomboid, and at other times that of the cube, which is the most perfect among the forms of this kind. In some cases the triangular prism is merely isoscele; in other cases it is equilateral; and in this last case, the relation between its height and the side of its base varies in both species. The tetrahedron undergoes analogous diversities.

There exist, however, forms of integrant molecules, as well as primitive forms which are common to several substances of diverse natures. For example, muriated soda and sulphuretted iron both have the cube as their primitive form. The regular octahedron is that of the ruby, and at the same time that of native bismuth. In this case the elementary molecules, although different in all respects, are so arranged that there results the same external configuration nearly; as in geometry, we may compose a square in several ways by assortments of figures which will differ from each other in the various squares. Besides, if observation has proved that mineral substances, distinct in their nature, sometimes exhibit secondary crystals of the same form, (for instance, regular hexahedral prisms with a diversity of structure which would suppose one in the forms of the molecules themselves) ought we to be surprised also to find in different species integrant molecules, the forms of which, similar externally, are owing to combinations of principles which cannot have any relation with each other? But it is worthy of attention that hitherto these forms common to several minerals were always those which have a remarkable character for simplicity

- Théorie de la Terre, par Lametherie, 2d edit. tome i. p. 35 et seq.

+ Observation only makes known the measurements of angles, and not the reference of dimensions; theory, however, furnishes us with data for determining the latter. 
and regularity, such as the cube, the regular octahedron, the dodecahedron with rhombous planes all equal and similar, Bec. These forms are kinds of limits which crystallization attains by different routes; while, with respect to other forms quite different from those limits, it has a single direction only which ends in any particular species of mineral.

Laws to which the Structure is suljected.-After having determined the primitive forms, and those of the integrant molecules, it remains to inquire for the laws pursued by these molecules in their arrangement, in order to produce these regular kinds of envelopes, which disguise one and the same primitive form in so many different ways.

Now observation shows that this surrounding matter is an assemblage of laminz, which, setting out from the primitive form, decrease in extent, both on all sides at once; and sometimes in certain particular parts only. This decrement is effected by regular subtractions of one or more rows of integrant molecules ; and the theory, in determining the number of these rows by means of calculation, succeeds in representing all the known results of crystallization, and even anticipates future discoveries indicating forms which, being still hypothetical only, may one day be presented to the inquiries of the naturalist.

Some very simple examples will serve for giving an idea of the laws to which the decrements in question are subjected.

[To be continued.]

$\mathrm{XV}$. The Bakerian Lecture. An Account of some new analytical Researches on the Nature of certain Bodies, particularly the Alkalies, Phosphorus, Sulphur, Carlonaceous Matter, and the Acids hitherto undecomposed; with some general Olservations on Chemical Theory. By HUMPH RY DAvY, Esq., Sec. R.S., F.R.S. Edin., and M.R.I.A.

[Continued from p. 19.]

IV. Analytical Experiments on Phosphorus.

$\mathrm{T}$

THE same analogies apply to phosphorus as to sulphur, and I have made a similar series of experiments on this inflammable substance.

Common electrical sparks, passed through phosphorus, did not evolve from it any permanent gas ; but when it was acted upon by the Voltaic electricity of the battery of five bundred plates in the same manner as sulphur, gas was produced in considerable quantities, and the phosphorus be- 